\title{
Scaling up the SIMPLE Design Model for Faculty Development: Lessons Learned
}

\section{Prof. Jill K Nelson, George Mason University}

Jill Nelson is an associate professor in the Department of Electrical and Computer Engineering at George Mason University. She earned a BS in Electrical Engineering and a BA in Economics from Rice University in 1998. She attended the University of Illinois at Urbana-Champaign for graduate study, earning an MS and PhD in Electrical Engineering in 2001 and 2005, respectively. Dr. Nelson's research focus is in statistical signal processing, specifically detection and estimation for applications in target tracking and physical layer communications. Her work on target detection and tracking is funded by the Office of Naval Research. Dr. Nelson is a 2010 recipient of the NSF CAREER Award. She is a member of Phi Beta Kappa, Tau Beta Pi, Eta Kappa Nu, and the IEEE Signal Processing, Communications, and Education Societies.

\section{Dr. Margret Hjalmarson, George Mason University}

Margret Hjalmarson is a Professor in the School of Education at George Mason University. Her research interests include engineering education, mathematics education, faculty development and mathematics teacher leadership.

\section{Prof. Anastasia P Samaras, George Mason University}

ANASTASIA P. SAMARAS is Professor of Education in the College of Education and Human Development at George Mason University, USA. She is an educational researcher and pedagogical scholar with signature work in self-study research methodology including co-editor of Polyvocal Professional Learning through Self-Study Research (2015) and author of Self-Study Teacher Research (2011) and lead editor of Learning Communities In Practice (2008). She is recipient of the Dissertation Research Award, University of Virginia, the Outstanding Scholar Award, University of Maryland, a Fulbright Scholar, and a Visiting Self-study Scholar. She served as chair of S-STEP from 2013-2015 and is a current Co-PI of two National Science Foundation (NSF) funded grants: Designing Teaching: Scaling up the SIMPLE Design Framework for Interactive Teaching Development and a research initiation grant: Student-directed differentiated learning in college-level engineering education. Her research centers on facilitating and studying her role in faculty development self-study collaboratives.

\section{Dr. Lori C. Bland, College of William and Mary}

Lori C. Bland, Ph.D., is a clinical associate professor of curriculum and research, and the Director of Curriculum, Center for Gifted Education at The College of William and Mary. She teaches courses in program evaluation, educational assessment, educational psychology, data-driven decision-making, and gifted education. Bland received her Ph.D. in Educational Psychology from the University of Virginia. Her current research focuses on assessing learning and professional outcomes in formal and informal STEM learning environments; how data are used to inform decision-making; and the uses of different research, evaluation, and assessment methods to solve educational problems. 


\title{
Scaling up the SIMPLE Design Model for Faculty Development: Lessons Learned
}

\begin{abstract}
This NSF DUE-funded project has supported the scaling and study of the SIMPLE model for faculty teaching development. The SIMPLE model provides a framework for ongoing teaching development in discipline-specific peer groups (SIMPLE groups) designed to support instructors as they learn about and try new research-supported interactive teaching strategies. Over the life of the project, SIMPLE groups have been active in six STEM departments at a large public research university. Studying the implementations in these six departments provided an opportunity to identify lessons learned for best practices in creation, leadership, and support of ongoing teaching development groups. This paper describes the lessons learned through this project and data that support them.
\end{abstract}

\section{Background and Motivation}

While existing literature supports the value of active and student-centered teaching practices for improving learning, retention, and engagement in post-secondary education, adoption of these practices has been slow in STEM disciplines [1, 2]. The primary goal of this NSF WIDER project was to study the impact of discipline-based faculty learning community model for teaching professional development. The SIMPLE model, which is described in further detail below, provides a structure for ongoing faculty-led teaching development. Designed to be adaptable and require little in the way of infrastructure, the SIMPLE model supports faculty as they learn about, implement, and revise evidence-based teaching strategies. Broadly speaking, these teaching strategies may be characterized as active, inquiry-based, and student/learnercentered. SIMPLE groups meet on a regular basis over at least one academic year to become familiar with new teaching strategies and to receive support and feedback as they work to innovate in their own teaching.

The structure of the SIMPLE model is motivated by literature on professional development in K12 and higher education, as well as by literature about faculty learning communities [3]. In particular, the model of an ongoing faculty development community to which participants bring their own challenges has been demonstrated to be effective in K-12 spaces [4]. Ongoing professional development (rather than a one-time intervention) has been shown to improve sustained impact in higher education $[5,6]$.

The guiding principles of the SIMPLE model, which were identified based on the results of a preliminary NSF project that studied STEM teaching development groups [7], are as follows: 
- Sustainable -- Groups are small (typically 4-6 people) to reduce logistical challenges and have low (or no) infrastructure costs and low barriers to entry, thereby facilitating longterm sustainability.

- Incremental change -- Participants are encouraged to undertake small, manageable changes in their teaching rather than engaging in a full overhaul their approach. The model emphasizes that a series of small changes leads to larger, sustainable change.

- Mentoring -- Groups are designed to provide mentoring from a group leader who has experience with student-centered teaching, as well as to encourage peer feedback and support across group members.

- People driven -- Participants bring their own teaching challenges to the group and identify potential ways to address those challenges from a menu of options introduced and discussed by the group.

- Learning Environments -- The teaching development emphasizes active and studentcentered teaching and learning while remaining open to the wide range of strategies that fall within that category.

\section{Project Structure}

The project was conducted at a large public university with very high research focus. SIMPLE teaching development groups were formed and studied in six departments, each with a unique culture and a unique set of teaching challenges. Each group had between 4 and 8 participants, in some cases with a few participants who came in and out over the active period of the group. Most groups included only faculty Instructors (both tenure-line and teaching-only), but two groups also included graduate teaching assistants among their members.

Each SIMPLE group had a leader who was selected based on their knowledge of and experience with active learning and student-centered teaching practices. The first round of group leaders went through a semester of training during which they met monthly to discuss recent literature in STEM education and their individual teaching change efforts. The discussion of their own efforts to adopt evidence-based teaching practices was intentionally in the style of a SIMPLE group, allowing them to experience participation before leading others. (There was some leader turnover, and later leaders did not engage in formal training.) Following training, group leaders formed their own SIMPLE groups within their home disciplines. For the first two years that discipline-based groups were active, group leaders continued to have monthly meetings, facilitated by the project leadership team, to share activities happening in their groups and to discuss challenges.

Group leaders took ownership of recruiting participants for their own groups. Leaders primarily used word of mouth for recruitment, extending invitations to those they thought would be interested and encouraging those people to spread the word. The six SIMPLE groups met 
regularly (anywhere from every two weeks to 2-3 times each semester) to learn about new teaching strategies and support each other in classroom efforts. Most groups scaffolded their discussion with resources such as How Learning Works [8] and Teaching and Learning STEM: A Practical Guide [9]. The time period over which the groups were active varied by group. Five groups met formally (in a structured, scheduled fashion) for at least two academic years, and one group continues to meet formally, now in its fifth year. Of the groups that no longer hold structured meetings, several members have shared that informal conversations about teaching among members continue.

\section{Data Collection and Methods}

Yearly individual interviews were conducted with both group leaders and participants over the first three years of the project; the quotes that appear in the Lessons Learned section were drawn from these interviews. A semi-structured protocol with a predefined set of questions was used for interviews, allowing the interviewer to ask follow-up questions to learn more from the interviewee as needed [10]. Interviewees were asked about what motivated them to participate in a SIMPLE group, what benefits the group provided for them, and what suggestions they had for improving the group structure and/or experience.

An inductive coding scheme with descriptive coding [11] was used to find common themes across the interview transcriptions. In this paper, we describe three themes that emerged across the groups during the first two years of group activity (when all groups were active). These themes, or lessons learned, emerged as group leaders and participants described their motivations for participating and their experience as part of a SIMPLE group.

\section{Lessons Learned}

Community matters. Many participants remarked on the importance of having a community in their department for discussing and supporting teaching. They noted that while nearly all faculty members in their department taught courses, there was no formal group for discussing teaching and that department-level discussion of teaching focused on the "what" (curriculum), not the "how". Finding a group of faculty in the same discipline who were also interested in improving their teaching provided a way to support their teaching change efforts. As one group leader said, "first of all, you are not the only person who struggles when the teaching dilemmas come up." As described by a group participant, "I think the idea is by talking, just by having this platform, it makes us feel that we are all on the same boat, because normally, before that I always felt - to me - I always felt that I am teaching alone." Another group member compared the SIMPLE group quite directly to a support group: "I called the group - informally - I called it Teachaholics Anonymous. We don't share enough of our ideas. Every...I would, I feel like...I will never be a hundred percent of the teacher I wanna be, and I think that's probably a good thing that I 
approach it that way. I'm always supposed to be evolving and getting better, and part of that is sharing our ideas, and we just don't do that. So I was attracted to joining that group because it was a forum for sharing ideas about teaching." Another participant noted the value of having a structure and visibility for the teaching work they were doing. "It's nice to be in a supportive atmosphere with other people that, you know, kind of care about teaching and improvement. It just makes you um, you know, makes you feel that any extra effort you put in is worth doing. And that you know you're being supported by, you know, the folks that might actually be interested or have some use for what you learn. I think, so it kinda gets a, just lends credibility, importance of, you know, doing this kind of work."

SIMPLE group members also mentioned the benefits of having accountability to a group as they worked to change their teaching. One participant described the SIMPLE group as similar to a gym buddy. "Committing to a change and feeling accountable for it, it's like going to the gym. You know, if you are going to the gym by yourself, you can say, "Well, I'm not gonna do it today," and nobody knows, but if you go to the gym with a friend, then when you say, "I'm not gonna do it today," then that friend's by themselves, or... So it's like, knowing that somebody else, you know, other people were also committed to uh, teaching excellence and that they're committed to making a change and uh, so I don't wanna go through and end up this point and not having had made a change when other people had."

Newer instructors identified the informal mentoring they received from more experienced faculty as a benefit of group participation. In describing why they participated in the SIMPLE group, one early-career instructor noted, "For me getting to listen to more-experienced instructors. To see that they were going through many of the same things I was going through. To see things they had tried and had worked to problems I was having that they had had in the past and had figured out a solution to."

Finally, participants frequently mentioned the value of SIMPLE groups for establishing longterm relationships with colleagues interested in teaching. While most groups stopped meeting formally after two or three years, the impact of these connections persisted. As described by a participant, "I've helped to foster my relationships with the people in that group, so now in dayto-day I can collaborate with those folks. Like one of them is my colleague across the hall. She's a part of our Teaching and Development group, and we've developed a great relationship, so she's one of my allies that I can go to. I know she gets it. I had another colleague who used to be diagonal from us - she was the same way. And she would tell me, "Here's what I struggle with, and I think you're really good at that. Here's how I can help you," and then I would go to her still and say, "OK, I know you respect my ideas, but I really struggle with this. Can I just talk it through with you?" And so, those relationships I think were fostered in our bigger group so I could do that in between." 
Discipline-focused groups have value. Analysis of data collected through this project also highlighted the value of discipline-based SIMPLE groups. Discipline-focused groups allow instructors to engage in deeper, informed discussions about the challenges presented by certain content and about how various courses in the curriculum are linked. Group members noted that other group members could quickly understand the nature of their challenges often had first-hand experience that was closely related, allowing them to share insights. One participant noted the value of learning from other instructors rather than from a book. In describing the benefits of participation in a SIMPLE group, they said, "Fresh ideas. Getting other people's perspectives on what I was doing, and then also just hearing people's ideas. And, having them - it was, so I read a bunch of books on teaching, right? But it was nicer to hear it from a person from a person who's done it, and so you can just ask, like, "Does that really work? ... It's nicer to have an interactive discussion than just reading about techniques in a book. Because sometimes it's hard to imagine actually implementing some of the techniques." Another participant mentioned how the discipline-specific nature of the group allowed them to discuss the details of adopting strategies from books on teaching. "I think that usually some of the sort of frequent topics that came up were, OK, here's this technique that's mentioned in the books. How applicable is it in our specific settings? Where we have forty to eighty undergraduate students, and we have this setting in which it's, they're technical questions. So, there's not as much room for interpretation as there would be in other disciplines, the humanities - like your art histories, English literature and poetry and so forth. So, if there's sort of a mention of like, 'Oh, this is a really good kind of activity to do in class, like how well does that scale to large setting? And if it's not immediately obvious, can we figure out a way where that might sort of work?"”

In several interviews, SIMPLE group participants identified times where they were able to discuss and receive feedback on situations or content specific to their courses. As described by a group participant, "It provided a formalized way of having these discussions on improving teaching and...the, you know, formalized groups were with people that were in my same field, so understood when I talked about my Statics class or I talked about my Structural Analysis class, every one of those people have taken those classes at some point." Another participant mentioned discussing both content and conduct concerns: "The expectations and honor code things were definitely brought up a lot. Like, clearly identifying expectations, and kind of preemptively dealing with those things that we know come up every semester - oh, everybody sucks at simplifying rational expressions. Let's post a video and give them a problem that they have to do before the class even starts, to turn in the first day of class." Yet another participant was able to draw on other group members' knowledge of lower-level courses when considering trying a new assignment: "I was thinking for the senior-level course I'm teaching, trying to add some paper reading elements into the course. ... I'm thinking to make it more interesting, for example, to let them present the paper, or form the groups to present the paper but I'm not too sure about whether it's too much for the undergraduate students. So I checked with the other teachers in the group, and some of them had experience with the undergraduate students 
presenting papers before in other classes - it's a different class - but they have done this, and so they assured me they can do this, even as undergraduate students. And they gave me very good suggestions about how to organize them into groups, how to let them, or at least allow them to choose the paper, but I have the final say to decide whether it's an appropriate paper or not, and how to do the presentation, get the evaluation, and things like that. Actually, after the group meeting, I added all those things to the teaching of this semester, and we will need to wait to see how that will go."

Group meetings need purpose and structure. While much of the feedback about leaders' and participants' experiences in a SIMPLE group was positive, group members also shared suggestions for improving the operation of the groups. A common theme across several interviewees was the need for a certain level of structure in group meetings. While the SIMPLE model is designed to be flexible and to adapt to the needs of each unique group, leaders and participants found it important to provide sufficient structure that each meeting had a clear purpose and focus. In the absence of a preset focus, group members noted that meetings fell into a stale pattern of broad discussions and common issues without focusing on possible solutions or new ideas. One participant said, "For me, it's the specific ideas. Here's something to try, or think about how it works, is what's interesting to me about this. A lot of the broader discussion I don't find as useful, that's not as well directed. I mean, sometimes the meetings got very a little bit off track in terms of, well, what's the point. Or how does this relate to active learning in other classroom, and what we are doing, and what's going on with the groups, and how we get more people trying these things, or where we should get some of these ideas as to what to try, or, you know, how do we get people going to see what this looks like or just to exchange these ideas and implement it, you know, would be useful."

Group leaders also identified the need for focus in group meetings. One commented on the need for group members to find value in each meeting in order to dedicate time to attending. "I would say the main issue is a slightly more focused approach to what we are doing, and again, it's about time. What do we have time for? ... Very focused topics I think are useful. This hearing for the fifth time, you know, oh I did this in my class, some of that was good the first time you hear, but then, how to get out and use it, you know. Should we send people for that class? That would be more useful than, oh OK, we go around again and everybody talks about sort of the same things again." Another leader gave the following advice: "If I had a piece of advice to give to other leaders, it would just be that you need to make sure folks know we're gonna meet for the following reason: to discuss this chapter, or to talk about this paper, or because so-and-so's gonna give this small presentation, or talk about this assignment - the structure that you think will be sort of informative. So, if each meeting has a somewhat well-defined purpose, I think that helps people to have buy in, it helps them to know how to prepare, or gauge their expectations of this as they come." Finally, a third group leader talked about how having a focus for each group meeting impacted participation. "I think having a specific topic per meeting with a reading is 
very critical. Because I think otherwise there always be this tendency of some people to not really participate at the meetings, they don't want to talk about things. I think it helped for us at least, it helped facilitate discussion each week, and what I saw is even the one person led the discussion, the people would jump in right off the bat and ... frankly, I can't think of a meeting we didn't go over, right? ... The times that we didn't do that - I didn't think those meetings went as well. The people just sat there and I didn't think the discussion was particularly rich."

\section{Conclusions}

SIMPLE group members (both leaders and participants) saw significant value in the creation of a space for learning about new teaching strategies and discussing teaching efforts. Creating groups focused on a particular discipline allowed for deeper discussion, as participants could understand details of others' courses and teaching efforts and hence could provide more targeted feedback. Feedback from both leaders and participants suggests that creating structure and purpose for group meetings is important to encouraging participation and maintaining the value of meetings over the long term. We continue to investigate the role of group leaders, content knowledge for teaching, and the value of different groups for their participants.

\section{References}

[1] L. Jamieson and J. Lohmann, Creating a culture for scholarly and systematic innovation in engineering education: Ensuring U.S. engineering has the right people with the right talent for a global society, American Society for Engineering Education, 2009.

[2] National Research Council, Discipline-based education research: Understanding and improving learning in undergraduate science and engineering, S.R. Singer, N.R. Nielsen, and H.A. Schweingruber, Eds., The National Academies Press, Washington, D.C., 2012.

[3] E. Wenger, Communities of Practice: Learning, Meaning, and Identity (1st pbk. ed.). New York: Cambridge University Press, 1999.

[4] S. Loucks-Horsley, K.E. Stiles, S. Mundry, N. Love, and P.W. Hewson, Designing Professional Development for Teachers of Science and Mathematics. Thousand Oaks, CA: Corwin Press, 2010.

[5] O.S. Anderson and C.J. Finelli, "A faculty learning community to improve teaching practices in large engineering courses: Lasting impacts," in Proceedings of the 121st ASEE Annual Conference, 2014.

[6] D. Zemke and S. Zemke, "Using a community of practice to diffuse instructional improvements into the classroom," in Proceedings of the 121st ASEE Annual Conference, 2014. 
[7] J.K. Nelson and M.A. Hjalmarson, "Faculty Development Groups for Interactive Teaching," Proceedings of the 122nd ASEE Annual Conference, 2015.

[8] S. Ambrose, How Learning Works: Seven Research-based Principles for Smart Teaching. Jossey-Bass, 2010.

[9] R.M. Felder and R. Brent, Teaching and Learning STEM: A Practical Guide. San Francisco, CA: Jossey-Bass, 2016.

[10] S.B. Merriam, Qualitative Research and Case Study Applications in Education. JosseyBass, 2001.

[11] J. Saldana, The Coding Manual for Qualitative Researchers (2nd Edition). Thousand Oaks, CA: Sage Publications, Inc., 2013.

\section{Acknowledgement}

This material is based upon work supported by the National Science Foundation under Grant No. 1347675 (DUE). Any opinions, findings, conclusions, or recommendations expressed in this material are those of the authors and do not necessarily reflect the views of the National Science Foundation. 